\title{
Research Paper \\ Role of Cognitive, Metacognitive, and Meta-Emotional Components in Prediction of Emotional Distress in Students
}

\author{
Pante'a Ahadian Fard ${ }^{1}$, Ali Asghar Asgharnejad Farid ${ }^{2 *}$, Fahimeh Fath-Ali Lavasani ${ }^{3}$, Ahmad Ashoori ${ }^{3}$
}

1. MSc., Department of Clinical Psychology, School of Behavioral Sciences and Mental Health (Tehran Institute of Psychiatry), Iran University of Medical Sciences, Tehran, Iran.

2. PhD in Psychology, Associate Professor, Department of Clinical Psychology, School of Behavioral Sciences and Mental Health (Tehran Institute of Psychiatry), Iran Univer sity of Medical Sciences, Tehran, Iran.

3. PhD in Psychology, Assistant Professor, Department of Clinical Psychology, School of Behavioral Sciences and Mental Health (Tehran Institute of Psychiatry), Iran University of Medical Sciences, Tehran, Iran.

\begin{tabular}{|l|l|l}
$\begin{array}{c}\text { Use your device to scan } \\
\text { and read the artice online }\end{array}$ \\
Cittation: Ahadian Fard P, Asgharnejad Farid AA, Fath-Ali Lavasani F, Ashoori A. [Role of Cognitive, Metacognitive, and \\
Meta-Emotional Components in Prediction of Emotional Distress in Students (Persian)]. Iranian Journal of Psychiatry and Clini- \\
cal Psychology. 2017; 23(2):178-191. https://doi.org/10.29252/NIRP.IJPCP.23.2.178 \\
dol": https://doi.org/10.29252/NIRP.IJPCP.23.2.178
\end{tabular}

Received: 16 Jul. 2016

Accepted: 10 Dec. 2016
Key words:

Emotions, Cogni-

tion, Metacognition

\section{A B S T R A C T}

Objectives The study aims to investigate the role of cognitive, metacognitive and meta-emotional components in predicting emotional distress in college students.

Methods This is a descriptive correlation study, and the study sample consisted of 400 college students who were selected by multistage cluster sampling. Data were obtained using Emotion Regulation Scale (ERS), Leahy Emotional Schemas Scale (LESS), Schema Questionnaire-Short Form (SQ-SF), Meta Cognition Questionnaire (MCQ-30), Mindful Awareness Assessment Scale (MAAS), and Acceptance and Commitment-II (AAQ-II).

Results Negative emotions like the acceptance and commitment, emotional self-awareness and mindfulness and positive emotions like acceptance of feelings, social isolation/alienation, vulnerability and trying to rationality can explain $42.1 \%$ of the inner inefficient emotion regulation methods. Negative emotions like higher values, mindfulness and acceptance of feelings and positive emotions like mistrust/ abuse and agreement can explain $27.2 \%$ of the external inefficient emotion regulation methods. Conclusion Levels of acceptance and practice, emotional self-awareness, mindfulness and acceptance of feeling less and social isolation/alienation, trying to rationality and vulnerability are higher as a result of the internal inefficient methods becoming higher. As levels of higher values, mindfulness and acceptance of feeling less and the mistrust/abuse and agreement are higher; as a result, the external inefficient methods becoming higher.

\section{Extended Abstract}

\section{Introduction}

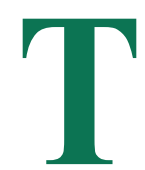

he ability to regulate excitements is an important growth task to maintain the inner balance of individuals, adaptive communication, and promoting mental health [1].
Some conceptualizations about the excitement regulation emphasize on control of emotional experiences, in particular, pretending to control negative emotions, and also the reduction of emotional excitement [2]. On the contrary, others emphasize the functional nature of excitement on excitement regulation and mention that the emotional regulation is not synonymous with emotional control. Therefore, it necessarily does not include immediate reduction of

* Corresponding Author:

Aliasghar Asgharnejad Farid, PhD

Address: Department of Clinical Psychology, School of Behavioral Sciences and Mental Health (Tehran Institute of Psychiatry), Iran University of Medical Sciences, Tehran, Iran.

Tel: +98 (21) 66506853

E-mail: asgharnejad.ali@gmail.com 
negative emotions. Research has been conducted on the importance of excitement in paving the path, continuity, resistance in treatment, quality of therapeutic relationship, and the type of selective interventions.

The present study attempted to identify emotional regulation problems and emotional distress in order to provide the basis for the use of appropriate therapies and techniques to increase the effectiveness of therapeutic interventions, prevent recurrence of disorder, resistance in treatment, and failure in the therapeutic relationship. Therefore, this study is trying to diagnose cognitive, metacognitive and metaexcitement components related to emotional distress.

\section{Method}

This study is a descriptive correlation study, and the statistical population is comprised of all Iranian medical students studying in 2014-15 academic year. In this research, multistage cluster sampling was used in a way that four colleges of Medical, Nursing, Midwifery, and Rehabilitation and Health were randomly selected among the faculties of Iran University of Medical Sciences. In every college, several classes have been randomly selected proportional to their students in each of the educational levels of that faculty. Then questionnaires were distributed randomly among these students. Among the participants, 223 people (57.5\%) were male and $165(42.5 \%)$ were female. Their mean age was 22.57 years, and the minimum age of the participants was 18 years and maximum age was 32 years. In regression analysis, for each predictor variable, there must be 10 subjects [19].
According to this article, the sample size should be at least 370 , but considering the size of society and falling rates, 400 people participated in the research. The variables studied have been listed as follows: 1. Emotional distress; 2. Emotional schemes; 3. Initial incompatibility schemas; 4. Metacognition; 5. Attention awareness; and 6. Commitment and acceptance. Results were analyzed using SPSS software version 22 and Pearson's two-variable correlation statistical strategies and step-by-step linear regression analysis.

\section{Results}

Table 1 shows the demographic characteristics of the participants in the study. Can variables such as emotional schemas, initial incompatibility scheme, metacognition, commitment and acceptance and attention awareness predict student emotional distress? To answer this question, a step-by-step linear regression method was used, and the two scales of intrinsic and extrinsic inefficient strategies were independently evaluated.

The dimensions of emotional schemas, initial incompatibility schema, acceptance and action, metacognition, and attention awareness were entered into the regression model in a step-by-step manner to predict the amount of intrinsic inefficient emotion regulation strategies. In the first step, acceptance and action entered the regression equation because of the highest correlation with highly intrinsic inefficient excitement regulation strategies. Next, the variables were split and analyzed, to make the maximum increase in the amount of the coefficient of determination $\left(\mathrm{R}^{2}\right)$. En-

Table 1. Demographic characteristics of participants

\begin{tabular}{|c|c|c|c|}
\hline \multicolumn{2}{|c|}{ Variables } & \multirow{2}{*}{$\begin{array}{c}\text { Frequency } \\
223\end{array}$} & \multirow{2}{*}{$\begin{array}{r}\text { Percen } \\
57.5\end{array}$} \\
\hline & Male & & \\
\hline Gender & & & \\
\hline & Female & 165 & 42.5 \\
\hline \multirow{4}{*}{ Education level } & Specialized PhD & 92 & 23.7 \\
\hline & General PhD & 40 & 10.3 \\
\hline & Master's degree & 86 & 2.22 \\
\hline & Bachelor degree & 170 & 43.8 \\
\hline \multirow{3}{*}{ Marital status } & Single & 315 & 81.2 \\
\hline & & & \\
\hline & Married & 73 & 18.8 \\
\hline \multirow{2}{*}{ Employment status } & Unemployed & 314 & 80.9 \\
\hline & Employed & 74 & 19.1 \\
\hline
\end{tabular}


try of variables continued to the point where the amount of significance of the model reached $95 \%$ and the error level reached $5 \%$.

Significance of $\mathrm{F}$ statistic $(\mathrm{F}=06.33)$ showed that regression of these variables (acceptance and action, emotional self-awareness, attention awareness, acceptance of feelings, social isolation/alienation, trying to be logical, vulnerability to the intensity of intrinsic excitement regulation strategies) was significant $(\mathrm{P}=0.0001)$.

There is a significant relationship between acceptance and action, emotional self-awareness, attention awareness, acceptance of feelings, social isolation/alienation, trying to be logical, vulnerability, and the intensity of intrinsic excitement regulation $(\mathrm{R}=0.649)$. In total, they accounted for $42.1 \%$ of the amount of intrinsic excitement regulation strategies $\left(\mathrm{R}^{2}=421\right)$. That way, the more was the extent of acceptance and action, emotional self-awareness, attention awareness, accepting fewer emotions, the higher was the level of social isolation/alienation, trying to be logical, vulnerability, and amount of intrinsic inefficient strategies.

\section{Discussion}

The results showed that there is a significant relationship between acceptance and action, emotional self-awareness, attention awareness, acceptance of feelings, social isolation/ alienation, trying to be logical, vulnerability, and the intensity of intrinsic excitement regulation strategies $(\mathrm{R}=0.649)$. In total, they accounted for $42.1 \%$ of the amount of intrinsic excitement regulation strategies $\left(\mathrm{R}^{2}=421\right)$. These results are in line with Schubler's view [37] that experience- and pattern-based avoidance in the person who does not want to be in contact with physical senses, excitements, thoughts or behavioral inclinations led to the use of internal and external inefficient methods. These results are also consistent with the model of Kuyken et al. [38], which mentioned that people have acceptance without judgment regarding their affairs. This refers to the awareness of perceptions, cognition schemas, emotions or body feelings, without judging or evaluating them being good or bad, true or false, healthy or unhealthy and important or insignificant. In this regard, Roemera [39] showed that the lower the level of awareness, the higher the amount of excitement problems. Also, Deplus [40] showed that attention awareness group interventions enhanced the ability to adjust the excitement in teenagers. Tang [41] presented a research where attention awareness as a mediating agent can increase self-control for excitement regulation.

The results showed that there is a significant relationship between higher values, mistrust/mistreatment, attention awareness, agreement, and acceptance of emotions with the severity of extrinsic excitement regulation strategies $(\mathrm{R}=0.522)$. In total, it accounted for $27.2 \%$ of the amount of extrinsic excitement regulation strategies $\left(\mathrm{R}^{2}=272\right)$. Due to the higher amount of higher values, attention awareness and acceptance of fewer emotions, mistrust/ill-treatment and agreement, the level of extrinsic excitement regulation strategies was also higher. In a study by Laura [42], it was shown that there was a significant relationship between awareness and reducing distress.

\section{Acknowledgments}

This research was extracted from the MSc. thesis of the first author, in the Department of Clinical Psychology, School of Behavioral Sciences and Mental Health (Tehran Institute of Psychiatry), Iran University of Medical Sciences, Tehran, Iran. Tehran Institute of Psychiatry has financially supported the present paper.

\section{Conflict of Interest}

The authors declared no conflicts of interest. 


\title{
نقش مؤلفهای شناختى، فراشناختى و فراهيجانى در بيشبينى يريشانى هيجانى در دانشجويان
}

\author{
•"انتهآ احديانفرد'، على اصغر اصغرنزاد فريدّ، فهيمه فتحلى لواسانى"، احمد عاشورى"
}

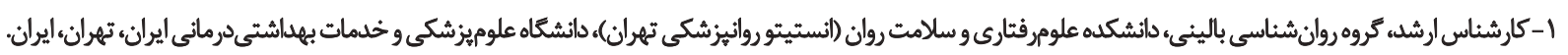

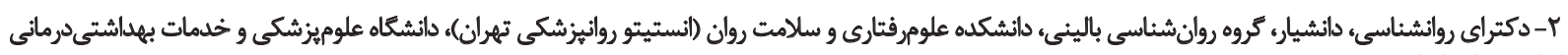
ايران، تهران، ايران.

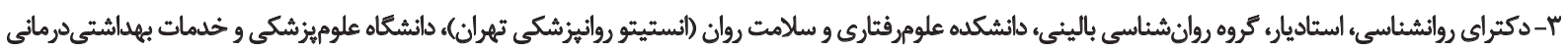
ايران، تهران، ايران.

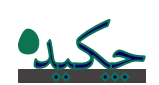

هداف يُؤهش حاضر باهدف ارزيابى ثأثير عوامل شناختى و فراشئاختى و فراهيجانى براي بيشيينى بريشانى هيجانى در بين دانشجويان انجام

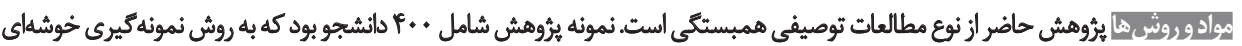

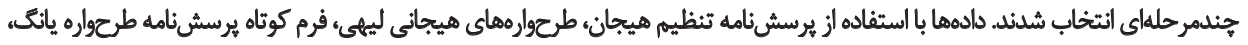

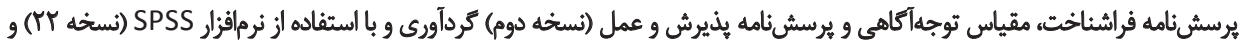

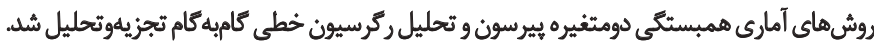

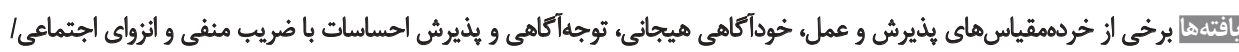

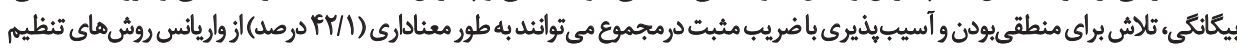

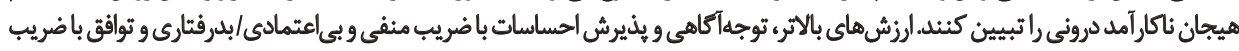

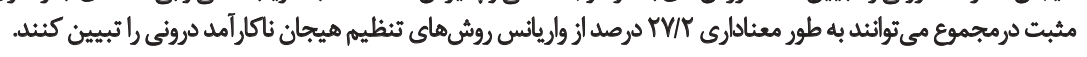

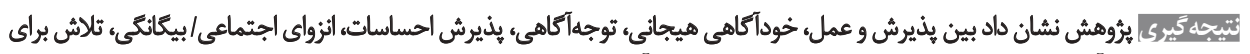

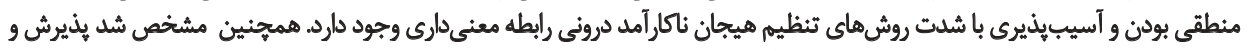

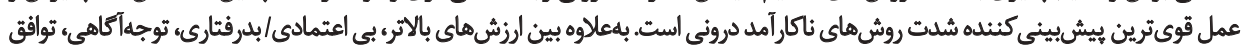

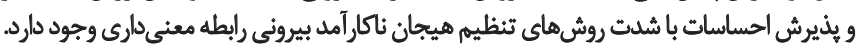

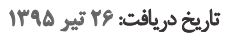

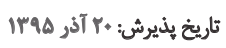

كليدوأرها:

هيجان، شناخت، فراشناخت

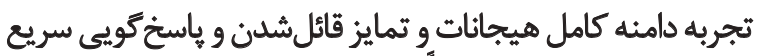

daves

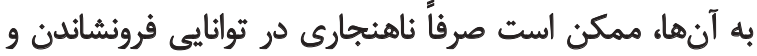

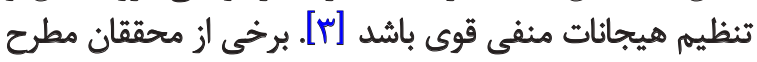

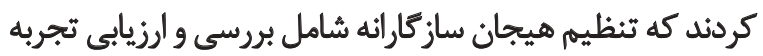

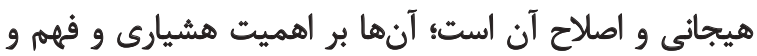

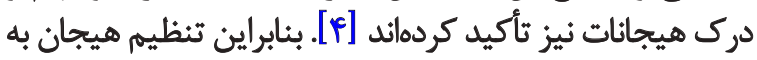

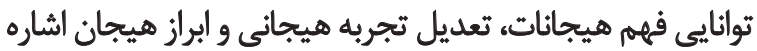

طبق مدل تنظيم هيجان كراس، تنظيم هيجان شامل همه

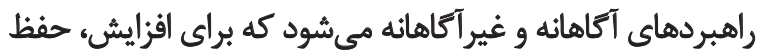

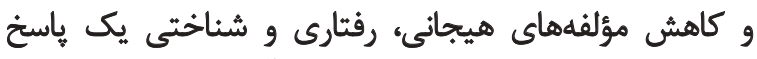

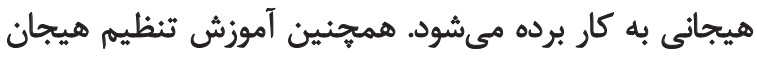

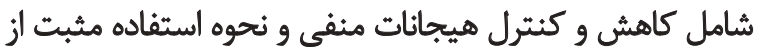

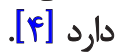

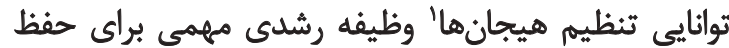

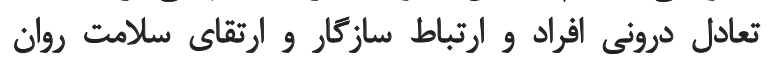

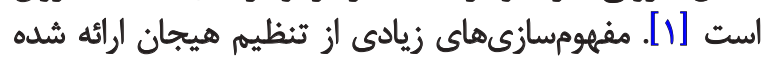

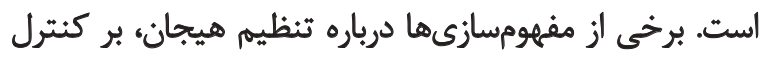

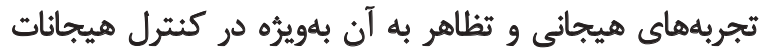

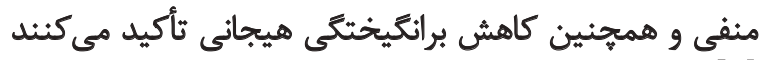

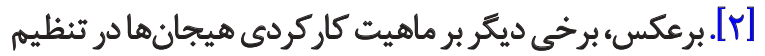

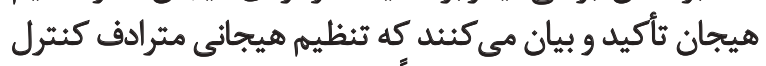

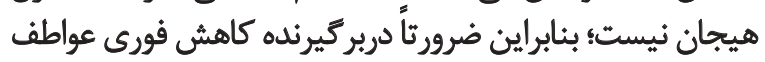

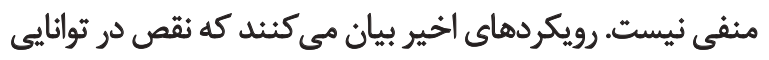

1. Emotion regulation

* نويسئده مسئول:

دكترعلى اصغر اصغرنزواد فريد

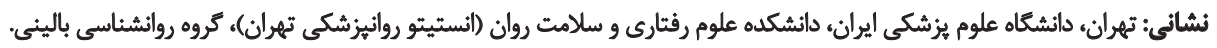

تلفن: يست الكترونيكي: تلعن: asgharnejad.ali@gmail.com 


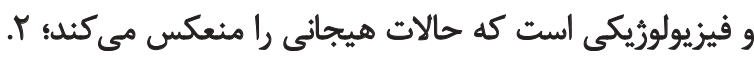

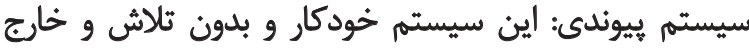

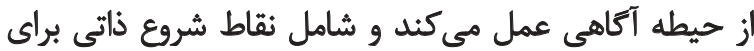

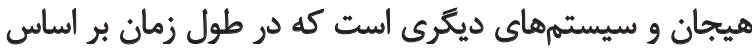

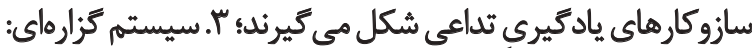

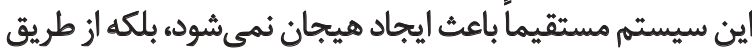

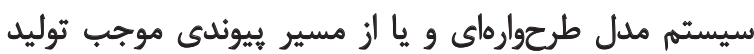

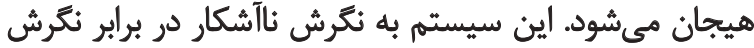

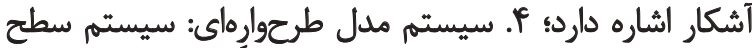

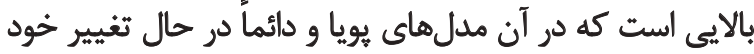

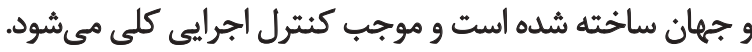

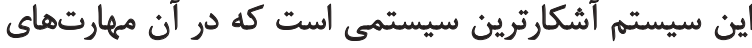

فراهيجانى مشاهده مي اشيود [9]

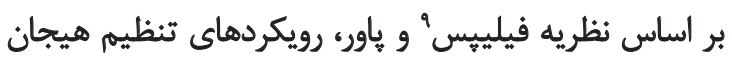

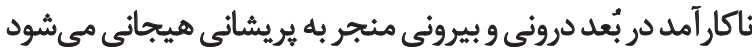

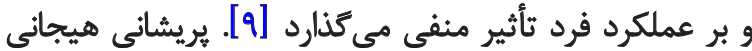

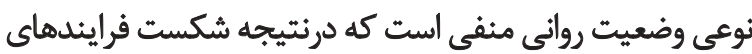

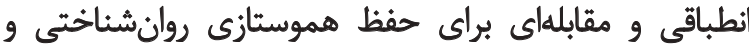

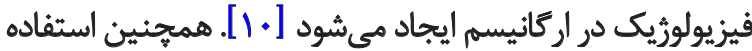

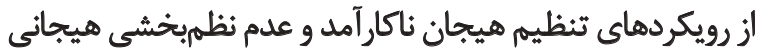

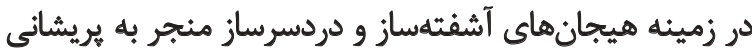

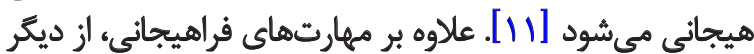

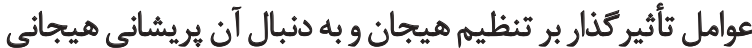

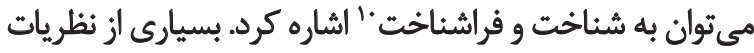

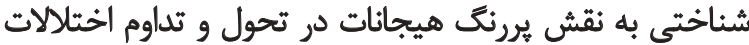

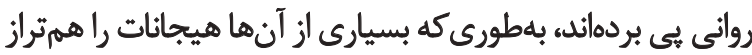

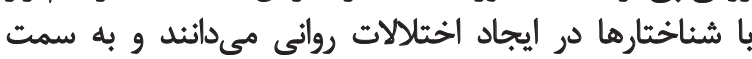

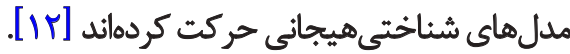

بنيان شناخت، طرحوارهها هستئد. فراشناخت، دانش يا يا فرايندى شناختى است كه در ارزيابي، بازبينى يا كنترل شناخ شناخت

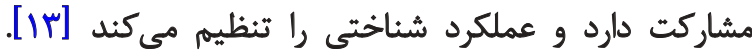

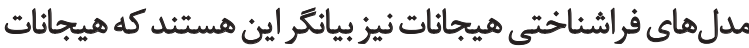

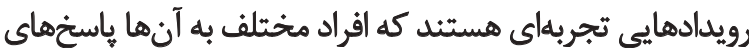

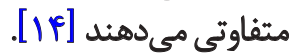

تنظيم هيجان عامل مهيمى در تعيين بهزيستى و عملكرد

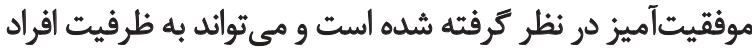

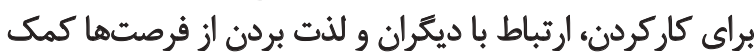

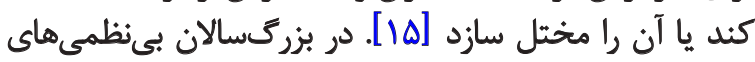

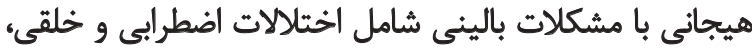

9. Philips

10. Metacognition
هيجانات است [هاء].

كراس ' [ع] در خلاصه جديدى كه از رويكردش ارائه كرده

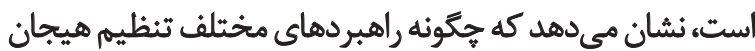

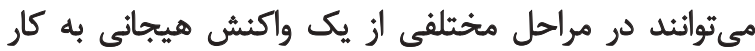

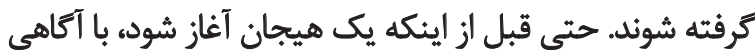

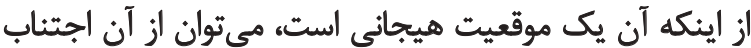

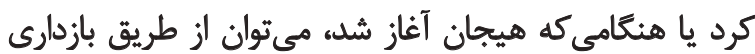

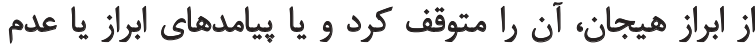

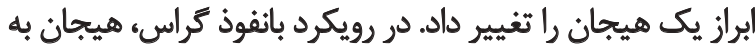

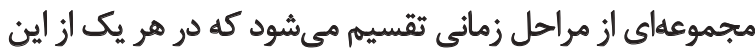

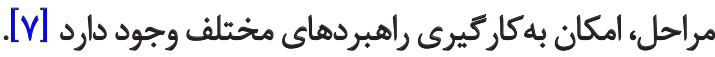
فيلييس و واور” [N] رويكردى كلنتر به تنظيم هيجان

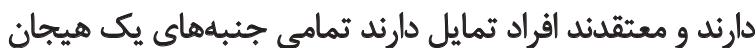

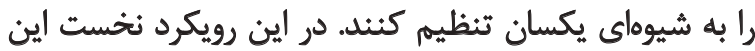

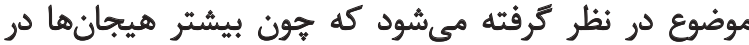

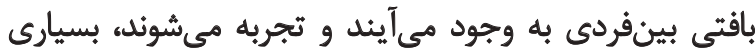

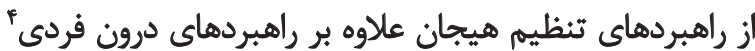

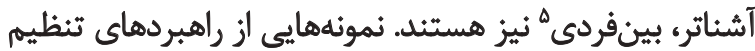

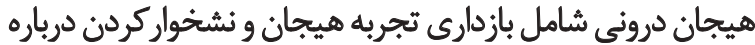

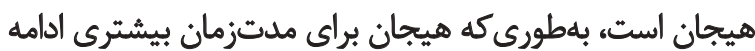

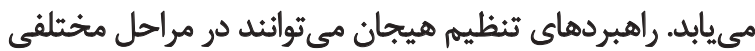

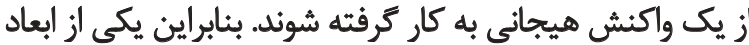

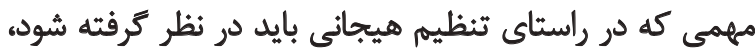

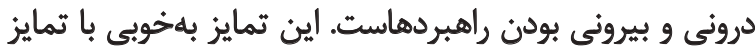

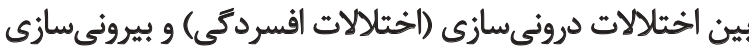
(اختلالات سلوك) در كودكان و نوجوانان هماهنك است.

بُعد ديكرى كه بر اساس آن مى توان تنظيم هيجان راسئ

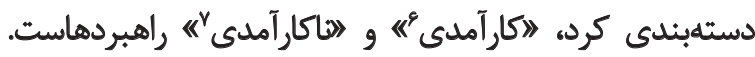

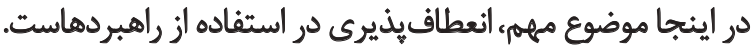

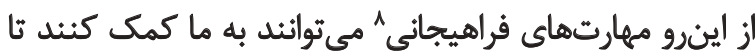

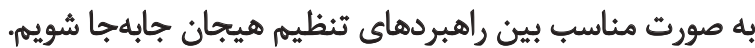

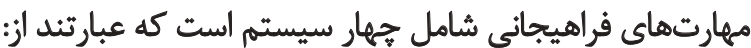

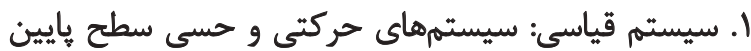

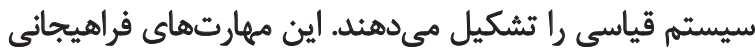

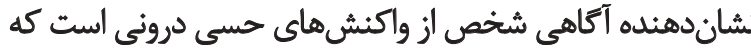

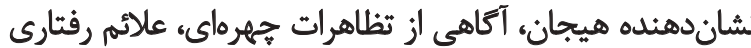

\section{Gross}

3. Power

4. Interpersonal

5. Intrapersonal

6. Functional

7. Dysfunctional

8. Meta emotional skills 
در هـر دانشكده بــه نسبت دانشجويان در هريك از مقاطع

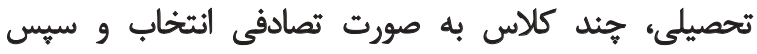

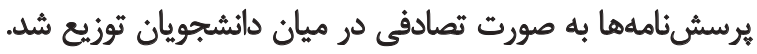

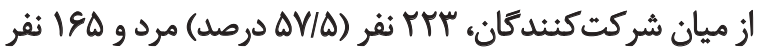
Fr/Q)

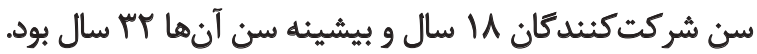

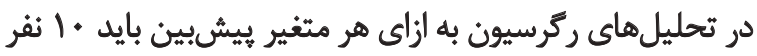

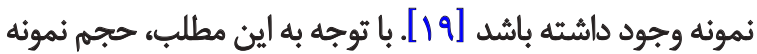

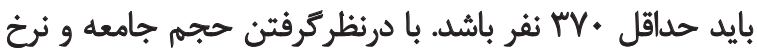

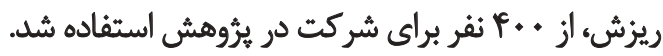

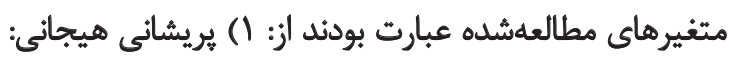

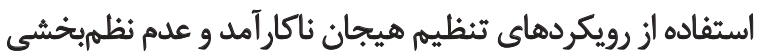

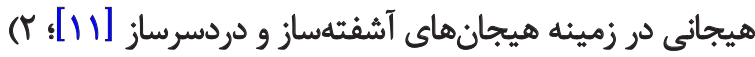

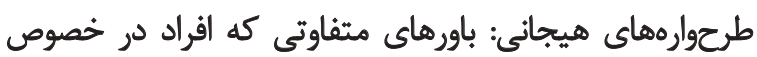

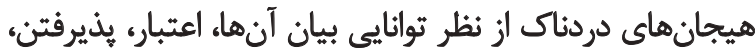

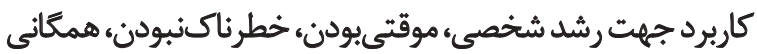

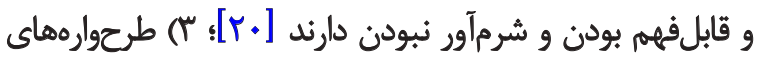

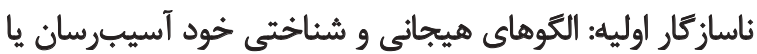
خودويرانترى كه شكل

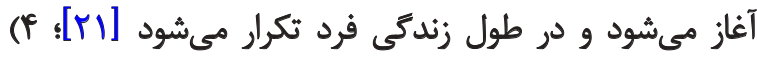

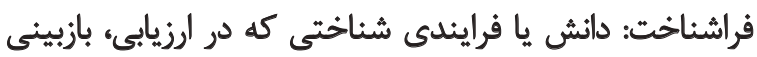

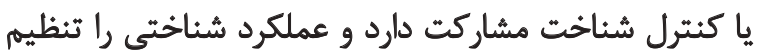

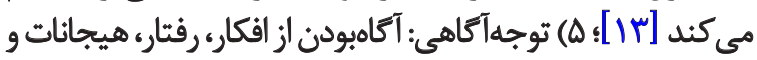

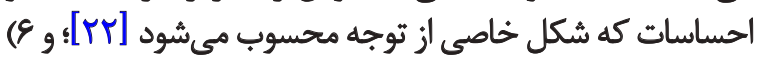

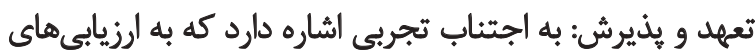

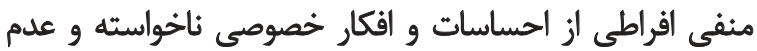

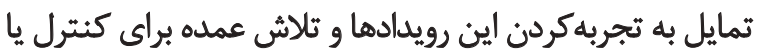
فرار از آنها اطلاق مي تشود اين روبادها إبزار يُوهنش

براى كردآورى دادهها يرسشنامههاى زير به كار برده شد:

\section{يرسش يامه تنظيم هيجان (ERS)}

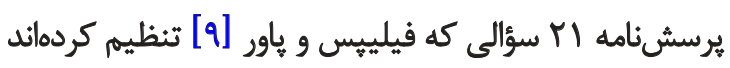

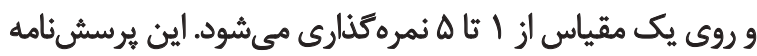

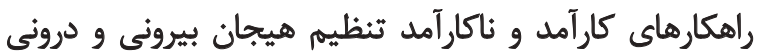

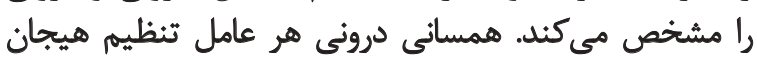

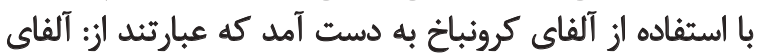

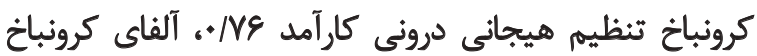

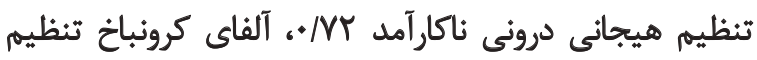

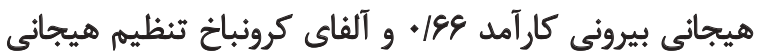

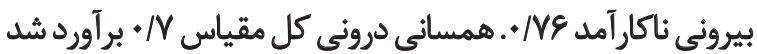

12. Emotion Regulation Scale (ERS)

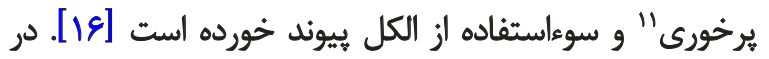

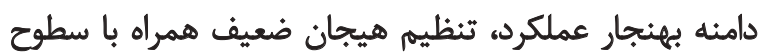

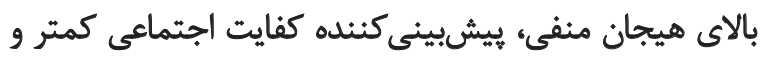

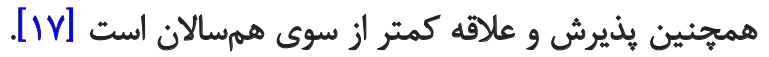

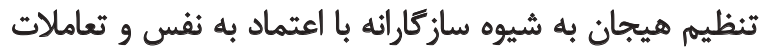

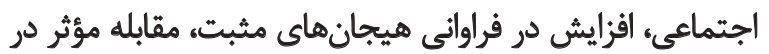

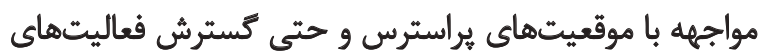

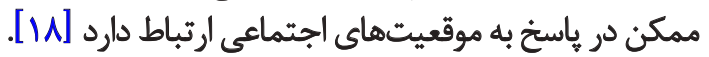
با توجه به تحقيقات انجامشده در داخل و خارج از كشور در

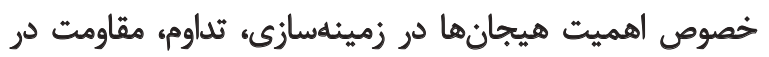

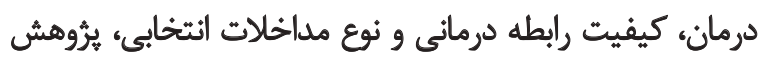

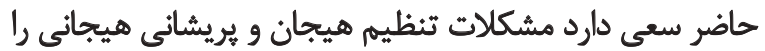

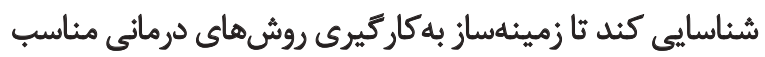

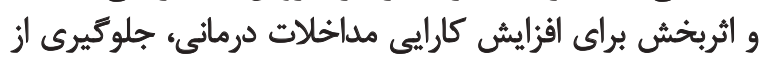

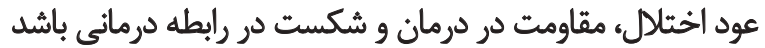

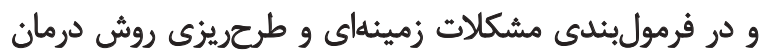

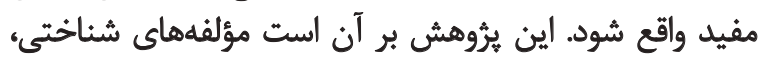

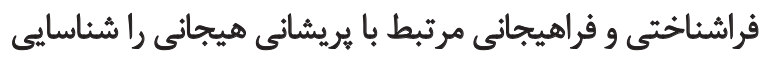

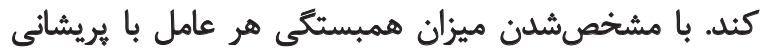

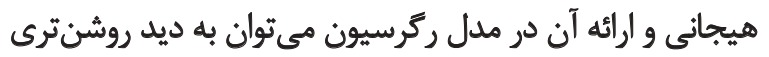

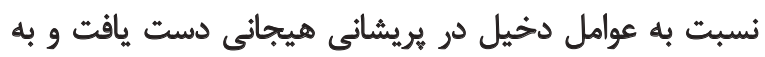

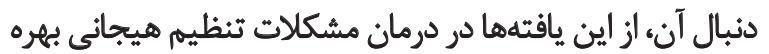

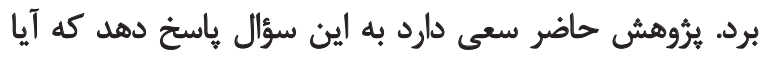

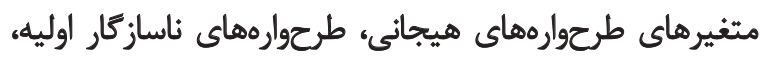

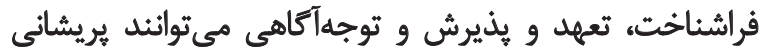
هيجانى دانشجويان را ييشبينى كنيند.

وازآنجايى كه هيجان و شناخت و رفتار با يكديكر در ارتباطاند

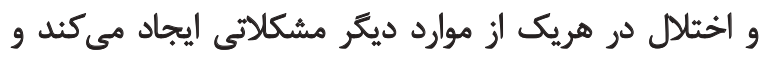

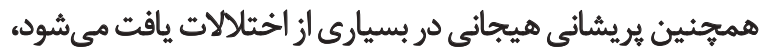

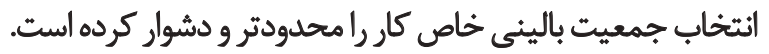

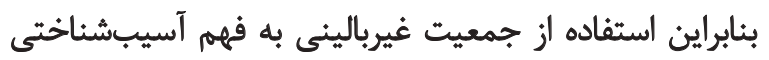

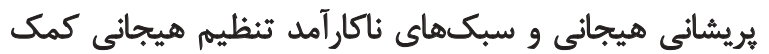
بسيارى مى كند.

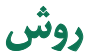

يُروهش حاضر از نوع توصيفى همبستّى است. جامعه آمارى

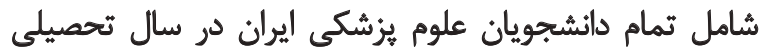

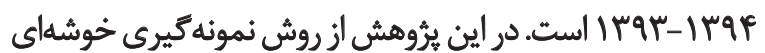

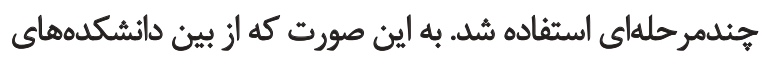

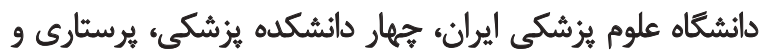

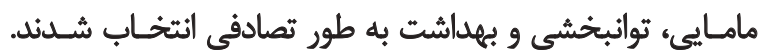




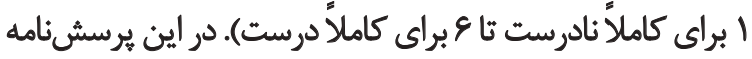

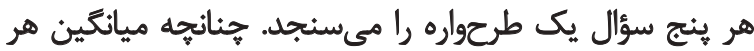

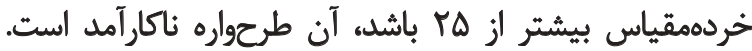

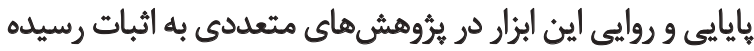

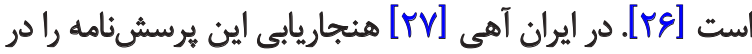

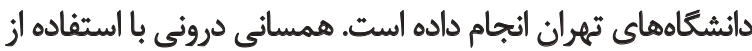

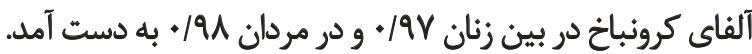

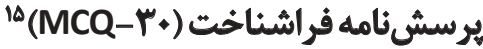

ولز و كاترايت هانتون اين يرسشنامه را ساختهاند كه • بـ آيتم

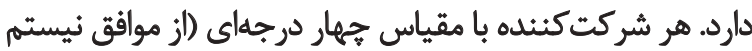

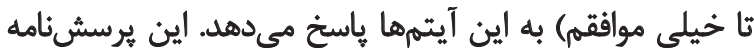

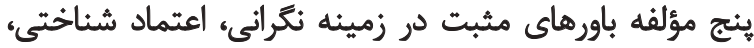

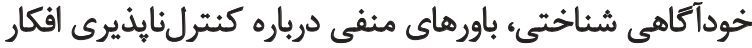

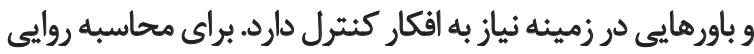

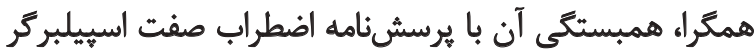

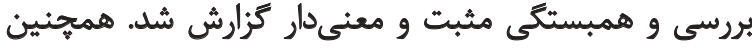

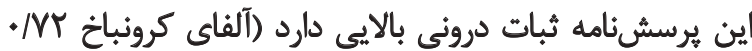

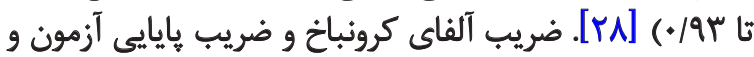

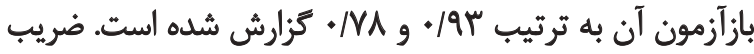

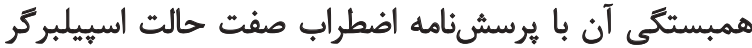

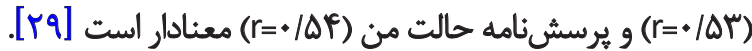

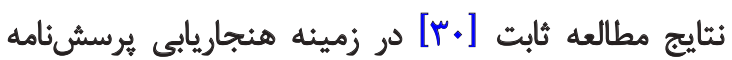

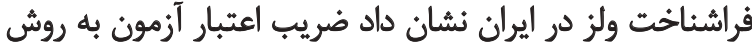

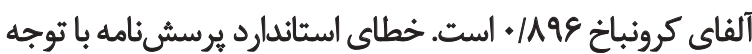

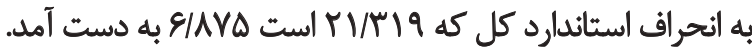

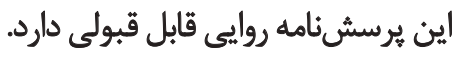

\section{مقياس توجه آكاهى (MAAS)}

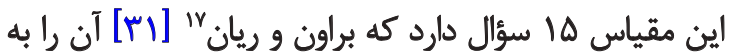

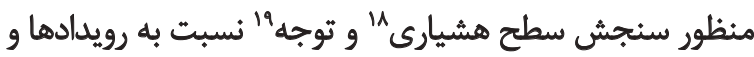

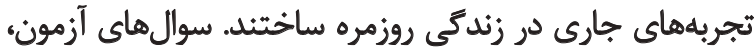

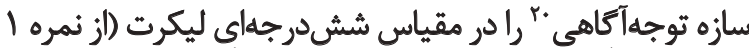

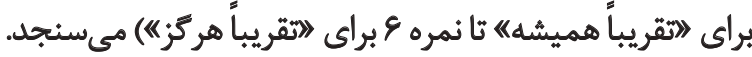

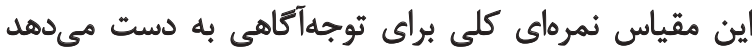

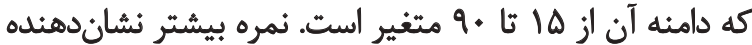

15. Metacognition Questionnaire-30 (MCQ-30)

16. Mindful Awareness Assessment Scale (MAAS)

17. Brown \& Ryan

18. Awareness

19. Attention

20. Mindfulness

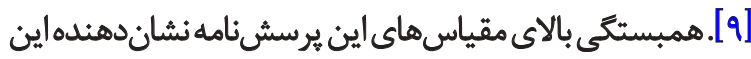

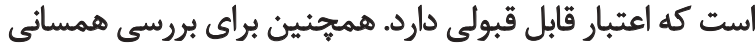

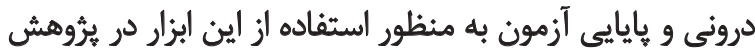

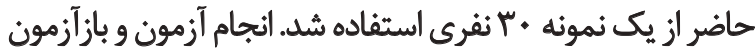

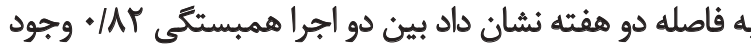

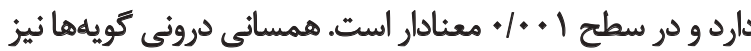

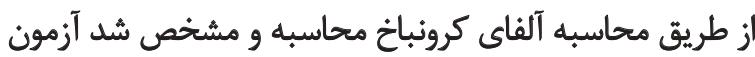

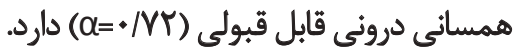

\section{"يرسش نامه طرحوارههاى هيجانى ليهى (LESS)}

ليهي [·r] يرسشنامه طرحوارههاي هيجانى را بر مبناي

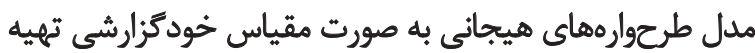

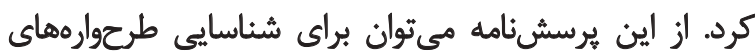

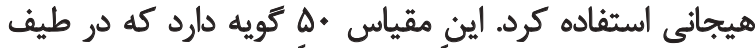

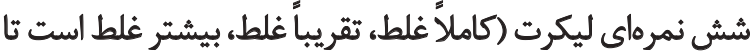

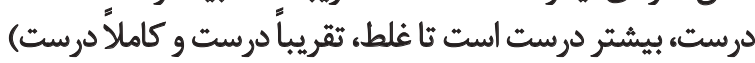

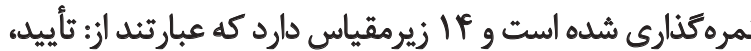

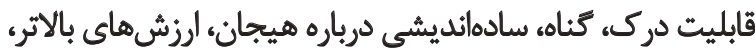

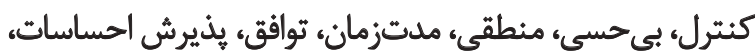

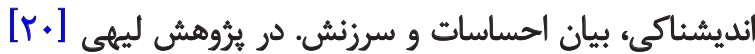

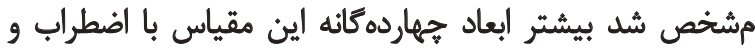

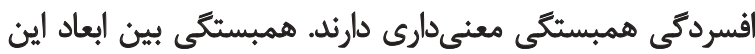

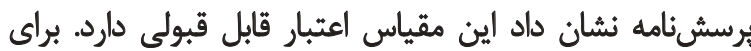

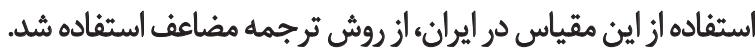

نتايج حاصل از بررسى عايايى نشان داد بايايى اين مقياس از

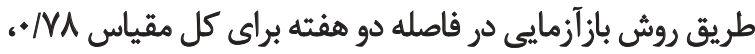

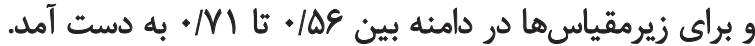

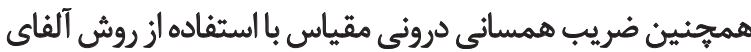

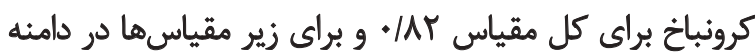

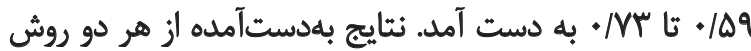

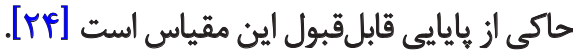

\section{If(SQ-SF) فرم كوثاه يرسش}

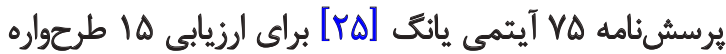

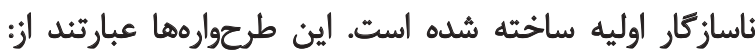

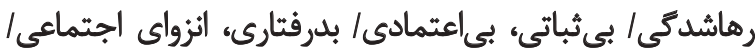

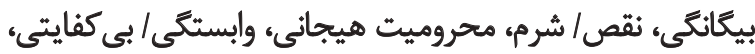

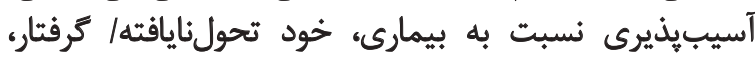

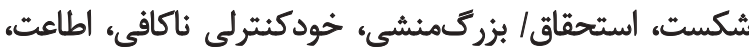

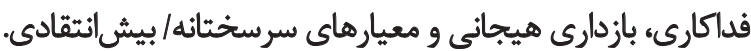

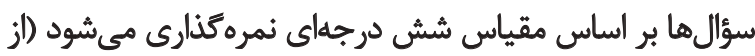

13. Leahy Emotional Schemas Scale

14. Young Schema Questionnaire-Short Form 
رانشان مي دهد. به نظر ميرسد يرسشنامه يذيرش و وعمل نسخه

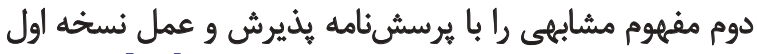

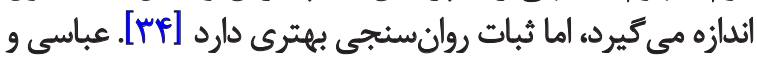

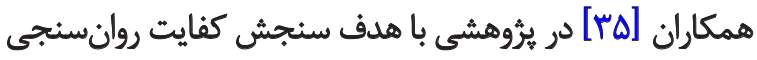

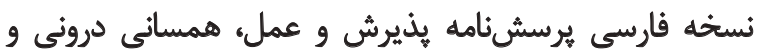

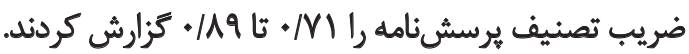

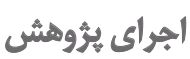

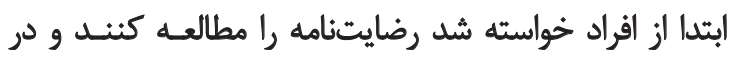

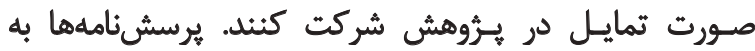

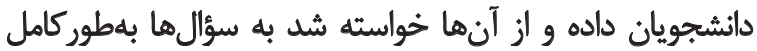

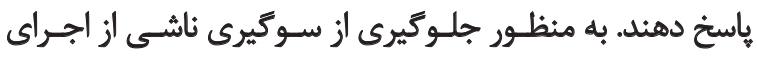

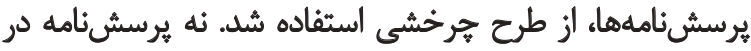

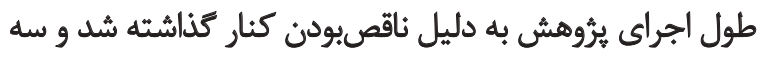

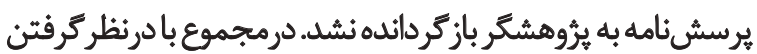

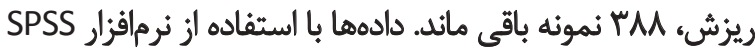

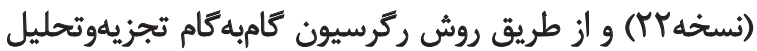

يافتهها

جدول شماره ا ويزگكىهاى جمعيث شناختى شركت كنيندكان

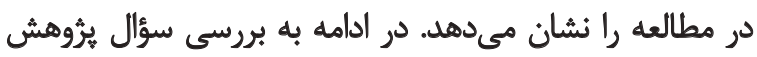

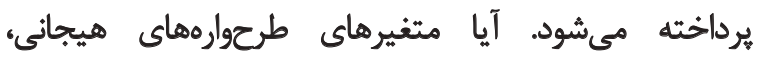

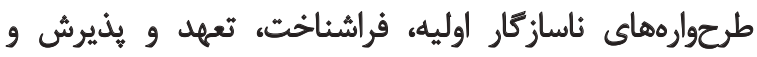

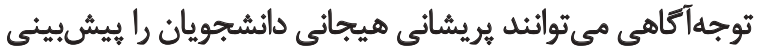

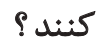

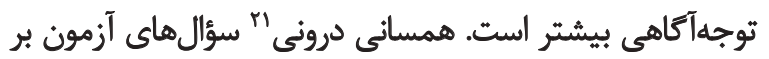

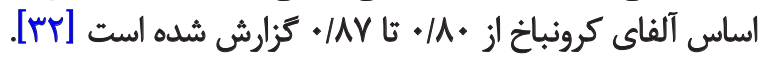

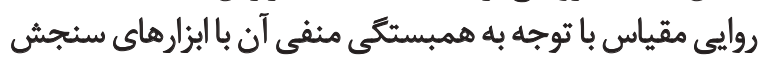

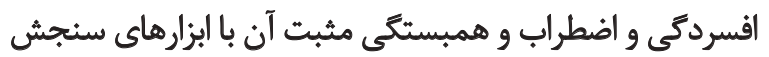

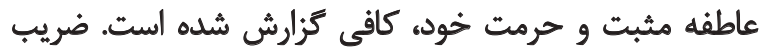

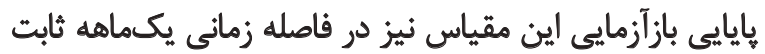

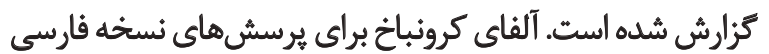

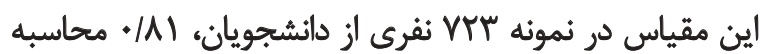

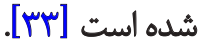

"يرسش هامه يذيرش و عمل (نسخه دوم) (AAQ-II)

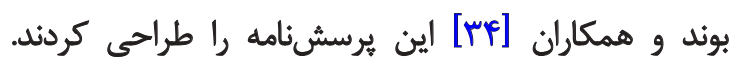

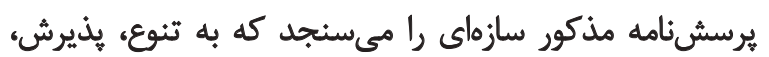

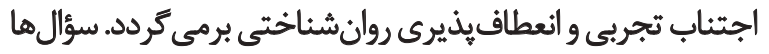

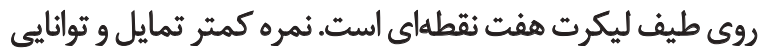

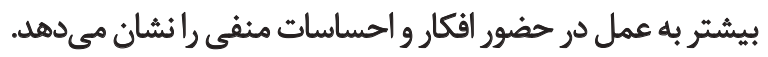

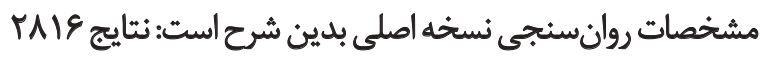

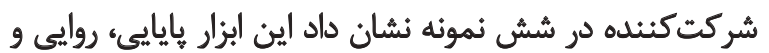

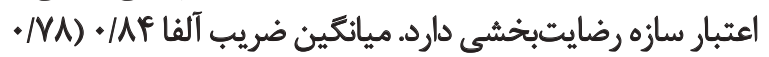

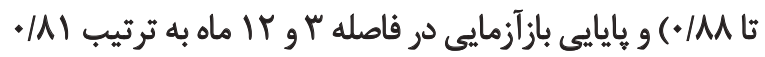

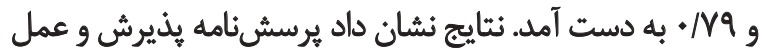

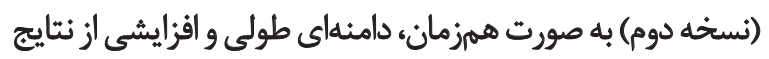

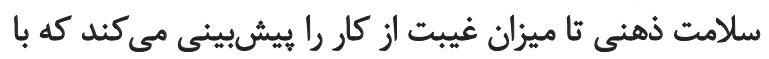

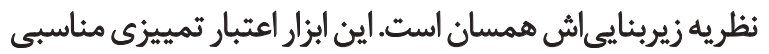

21. Internal consistency

22. Acceptance \& Action Questionnaire (AAQ-II)

جدول ا.ويئكىهاى جمعيتشناختى شركتكنيدكان

\begin{tabular}{|c|c|c|c|}
\hline درصد & فراوانى & \multicolumn{2}{|c|}{ متغيرها } \\
\hline$\Delta V / \Delta$ & TM & 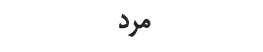 & \multirow{2}{*}{ جنس } \\
\hline$\mu / \Delta$ & $19 \Delta$ & زن & \\
\hline$\pi / N$ & qr & دكتراى تخصصى (PhD) & \multirow{4}{*}{ مقطع تحصيلى } \\
\hline $1+/ 4$ & f. & دكتراى عمومى (يزشكى & \\
\hline$r / T r$ & $N$ & كارشناسى ارشد & \\
\hline$\mu r / \Lambda$ & IV. & كارشتاسى & \\
\hline $11 / r$ & Ma & ل مجردر & \multirow{2}{*}{ وضعيت تأهل } \\
\hline WA & $n$ & متأهل & \\
\hline $1 . / 9$ & mif & بلدون شغل & \multirow{2}{*}{ وضعيت اشتعال داتشجوي } \\
\hline $\mid 9 /$ & $n$ & شاغل & \\
\hline
\end{tabular}

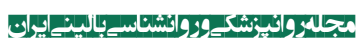


جدول r. خلاصه مدل بيشبينى شدت روش هاى ناكارآمد درونى

\begin{tabular}{|c|c|c|c|c|c|}
\hline Std.Error & $\mathbf{R}^{2} \mathbf{a d j}$ & $\mathbf{R}^{2}$ & $\mathbf{R}$ & مثغير ييشبين & 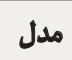 \\
\hline$T / N+$. &.$/ r a$ & . / Tar & .10 .4 & يذيرش و عمل & كام 1 \\
\hline T/OAP & $\cdot$ MIr & 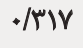 & . $10 \mathrm{E}$ & لئيرش و عمل، خودآكاهي هيجانى & Lام \\
\hline$r / \Delta \cdot Y$ &.$/ T \Delta$ &. & $.18 \cdot Y$ & يذيرش و عمل، خودآكاهى هيجانى، توجلأكاهي & كام \\
\hline T/Fe. &.$/ M W$ & . Tras &. & يذيرش و عمل، خودآَاهى هيجانى، توجهآكاهى، يذيرش احساسات & Fle \\
\hline the &.$/$ pqf & $\cdot / 4 \cdot 4$ & .1970 & 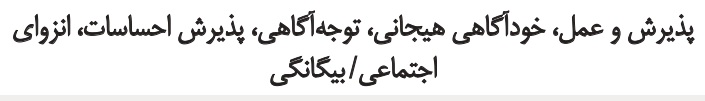 & كام \\
\hline$r / 4 I I$ & $\cdot / t+r$ & $+/ 414$ & $.194 \pi$ & 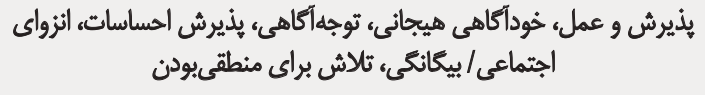 & كام \\
\hline r/maY & $.18 \cdot 9$ & . & .1979 & 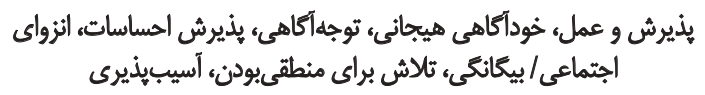 & $V^{8}$ \\
\hline
\end{tabular}

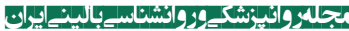

هيس از تفكيك متغير مقدم بر آن، وارد ثحليل شدند ثا باعث

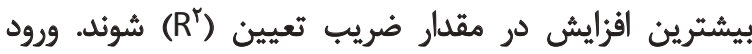

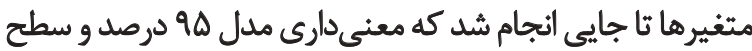

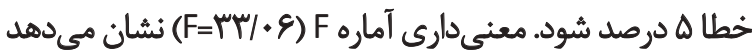

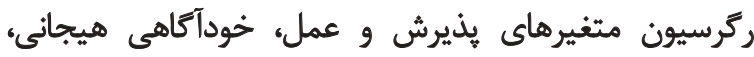

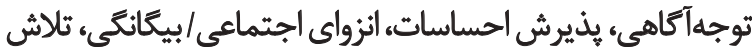

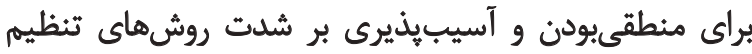

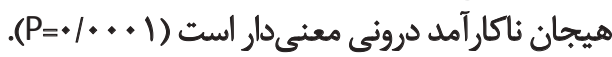

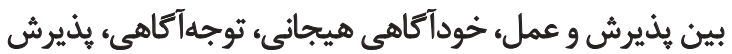

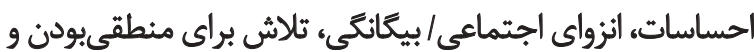

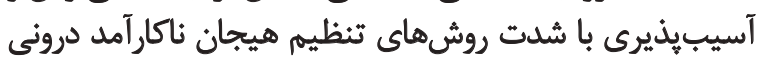

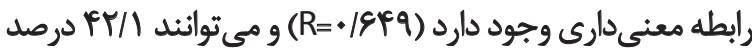

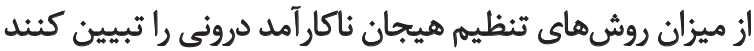

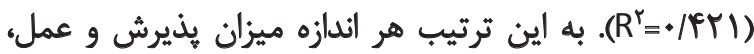

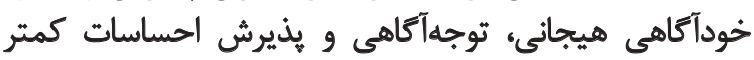

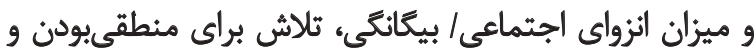

براي بررسى اين سؤال از روش ركرسيون خطى به روش إش

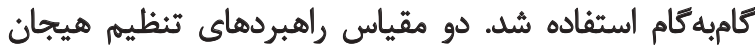

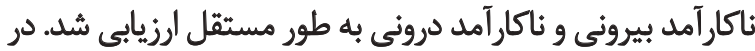

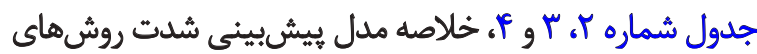

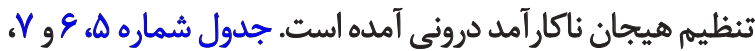

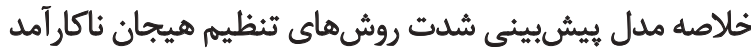

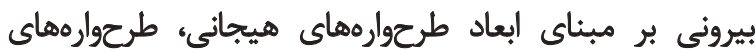

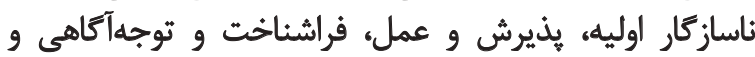

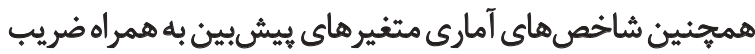

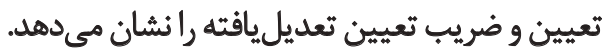

ابعاد طرحوارههاي هيجاني، طرحوارههاي ناسازكار اوليه؛

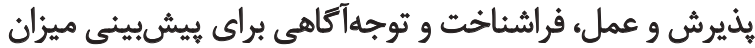

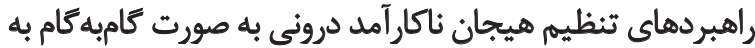

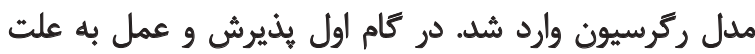

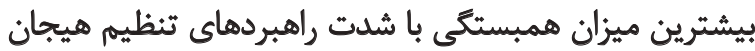

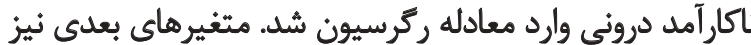

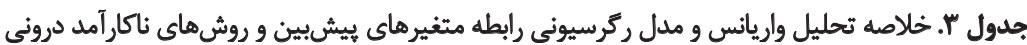

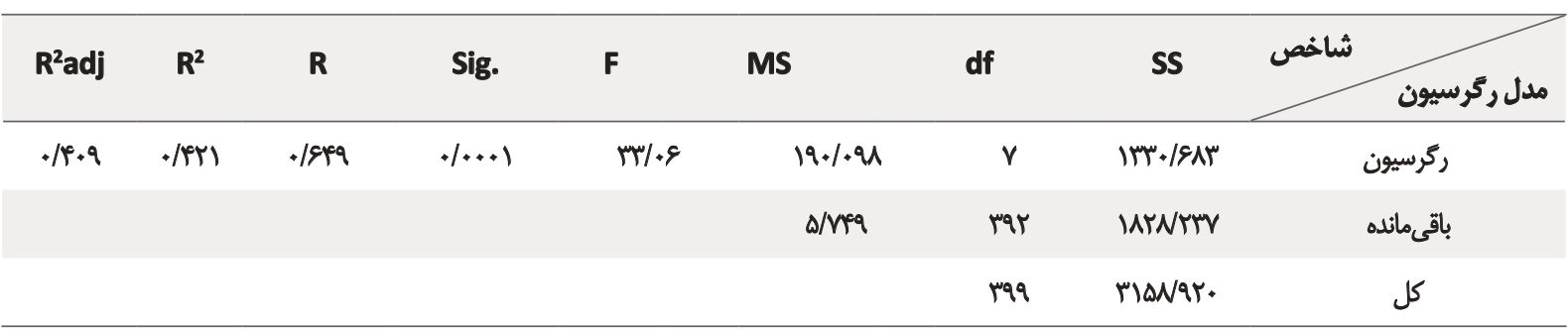


جدول F. شاخصهاي آمارى ركرسيون متغيرهاي بيشبين شدت روشهاي ناكارآمد درونى

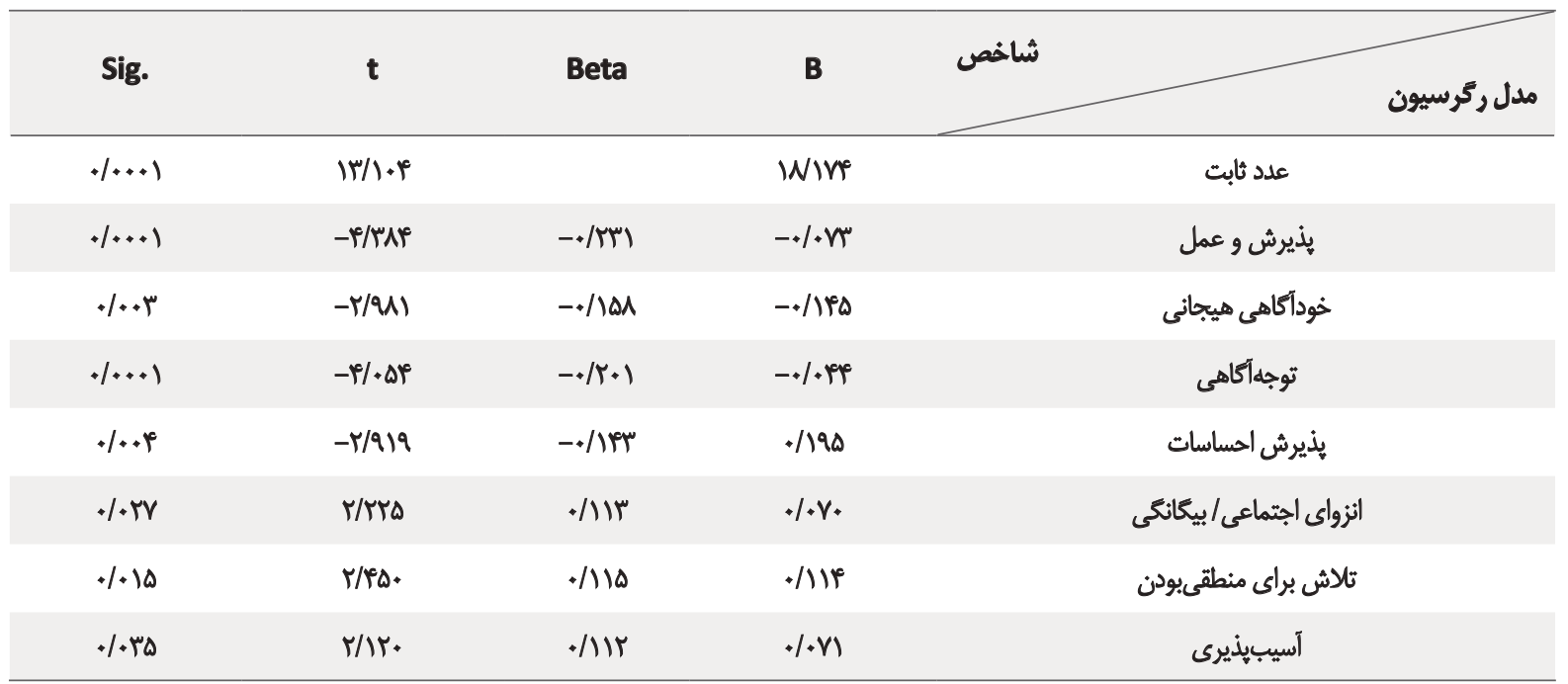

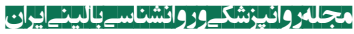

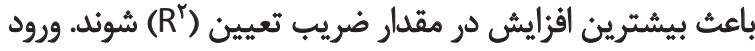

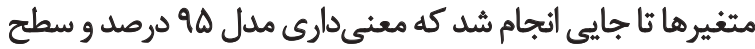

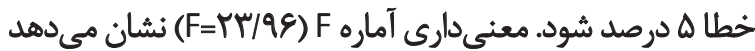

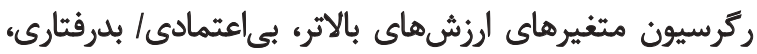

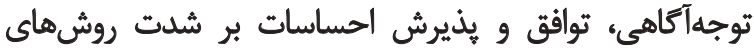

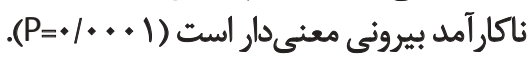

بين ارزشهاى بالاتر، بى اعتمادى/ بدرفتارى، توجهآكاهي،

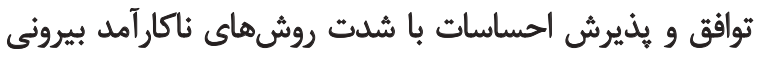

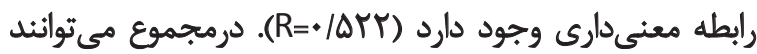

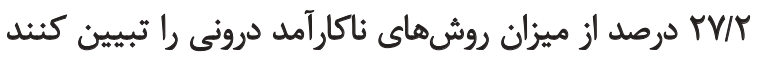

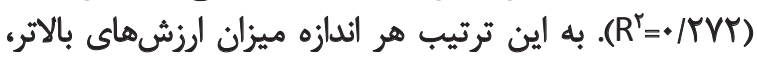

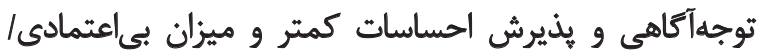

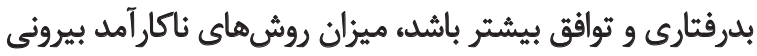

$$
\text { بيشتر است. }
$$

اين مدل كه فرمول كلى آن در زير ارائه شده است مي تواند

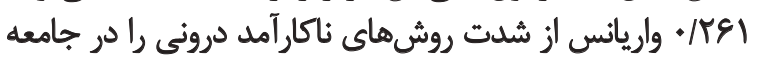

آسيب يذيرى بيشتر باشد، ميزان روش هاى ناكار آمد درونى بيشتر

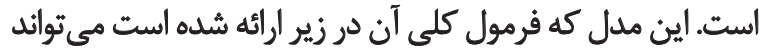

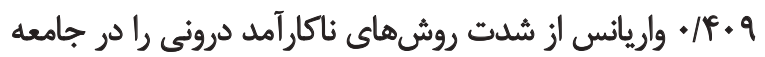

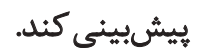

روشهاى ناكارآمد درونى = (يذيرش و عمل) - INIVF

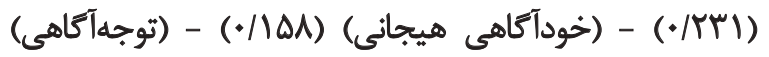

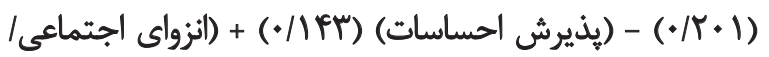

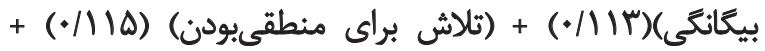

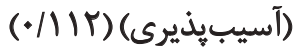

ابعاد طرحوارههاى هيجانى، طرحوارههاى ناسازئار اوليه،

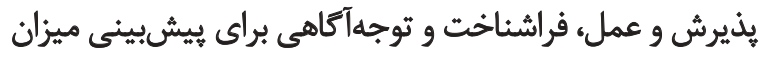

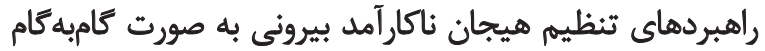

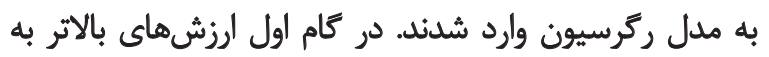

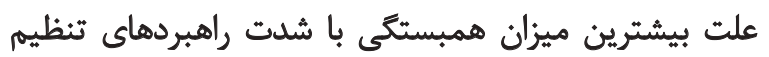

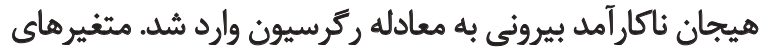

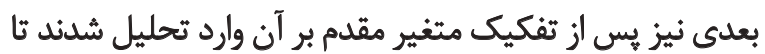

جدول ه. خلاصه مدل جيشبينى شدت روش هاى ناكارآمد بيرونى

\begin{tabular}{|c|c|c|c|c|c|}
\hline Std.Error & $\mathbf{R}^{\mathbf{2} a d j}$ & $\mathbf{R}^{\mathbf{2}}$ & $\mathbf{R}$ & مثغير بيشبين & 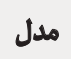 \\
\hline MIMY & . MFa &.$/ 101$ &.$/$ HA & الرزش هاي بالاتير & كام ام 1 \\
\hline$M / \cdot \Delta \Lambda$ &.$/ M I T$ &.$/ \pi M$ & ./eer & ارزش هاي بالاتر، بى اعتمادى بلدرفتارى & to lo \\
\hline$r \%$ &.$/ T F$ &.$/$ MP & $\cdot /$ pqr & 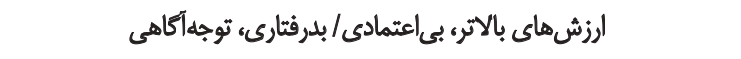 & rpls \\
\hline r/MA &.$/ T Q$ &.$/ 48$ &.$|\Delta|$ & ارزش هاى بالاتر، بي اعتمادى/ بلرفتارى، توجهآكاهى، توافق & Pl \\
\hline thep &.$/ M E 1$ &.$/ 4 r$ & . IOYY & ارزش هاي بالاتر، بيىاعتمادى بلدرفتارى، توجهآكاهي، ثوافق، يذيرش احساسات & كام هام \\
\hline
\end{tabular}

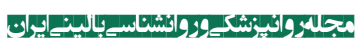


جدول و خلاصه تحليل واريانس و مدل ركرسيونى رابطه متغيرهاى يُيشبين و روشهاى ناكارآمد بيرونى

\begin{tabular}{|c|c|c|c|c|c|c|c|c|}
\hline $\mathbf{R}^{2} \mathbf{a d} \mathbf{j}$ & $\mathbf{R}^{2}$ & $\mathbf{R}$ & Sig. & $\mathbf{F}$ & MS & df & SS & \\
\hline \multirow[t]{3}{*}{. REI } & $\cdot \pi r$ & / /OYY & $.1 . .+1$ & $\mathrm{~m} / \mathrm{qs}$ & $M \cdot / P M$ & $v$ & $1 . \Delta Y / P T q$ & , ركرسيون \\
\hline & & & & & NVN & mar & YAIV/AQ & باقى مانده \\
\hline & & & & & & 199 & HAeF/.rV & كل \\
\hline
\end{tabular}

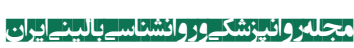

جدول V. شاخص هاى آمارى ركرسيون متغيرهاى ييشبين شدت روشهاى ناكارآمد بيرونى

\begin{tabular}{|c|c|c|c|c|c|}
\hline Sig. & $t$ & Beta & B & & مدل ركّرسيون \\
\hline$+1++\infty 1$ & $\mid r / \cdot g r$ & & $\mid \Delta / \Delta \Delta V$ & علد ثابت & \\
\hline$+1+++1$ & $-F / A W$ & $-+/$ TAq & $-+/ 4+r$ & ارزش هاى بالاتر & \\
\hline$+1+.+1$ & Pl.ep & $\cdot r+\Delta$ & . MFP & بىاعتمادي/بلرفتتارى & \\
\hline $.1 .+1$ & $-r / r \Delta A$ & $-\cdot$ MAY & $-\cdot 1+\infty i$ & توجهآكاهى & \\
\hline $.1+V$ & $r / e q p$ & .1149 & - MFA & توافق & \\
\hline$+1+19$ & $-r / H E V$ & $-+/ N T$ & $-+/ A Y V$ & يُّيرش احساسات & \\
\hline
\end{tabular}

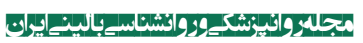

تماس با حسهاى بلدنى، هيجانات، افكار و يا تمايلات رفتارى

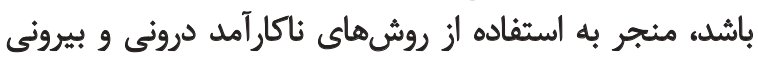

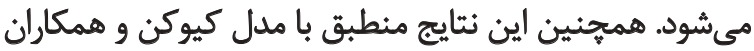

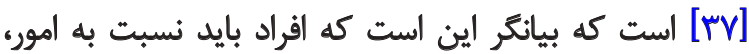

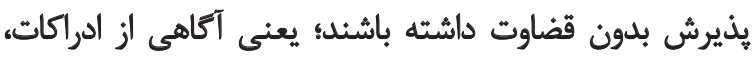

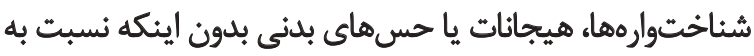

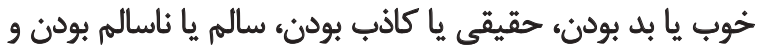

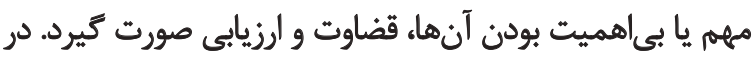

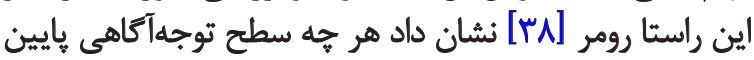

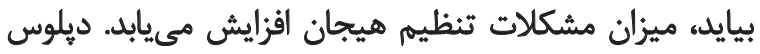

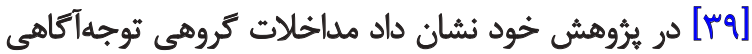

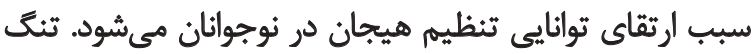

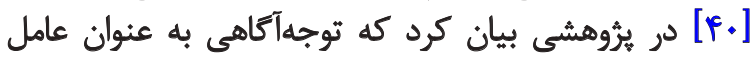

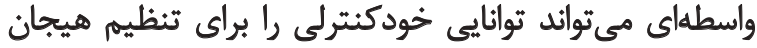

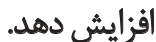

\section{نتيجهُمَيرى}

نتايج هيروهش حاضر نشان داد بين ارزشهائ بالاتر،

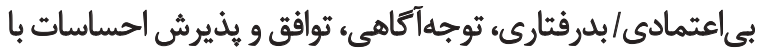

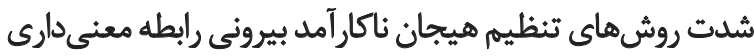

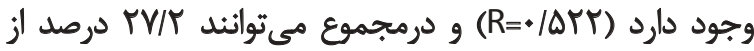

بيشيش:بيني كند.

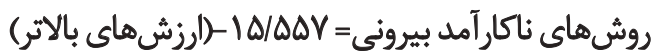

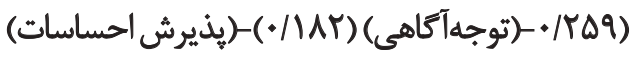

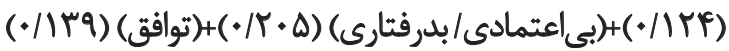

ث

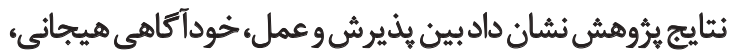

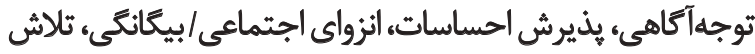

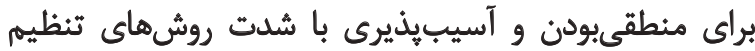

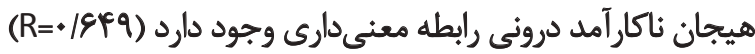

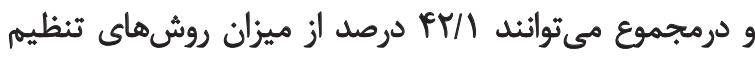

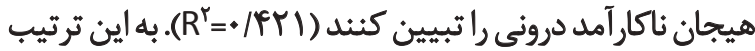

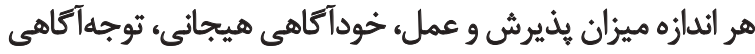

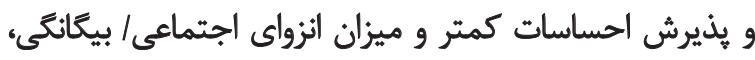

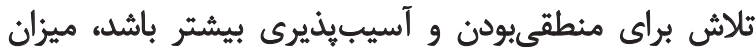

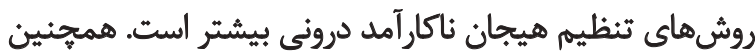

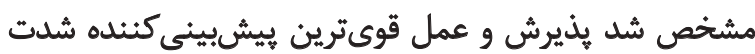

$$
\text { روشهاي ناكار آمد درونى است وشئ }
$$

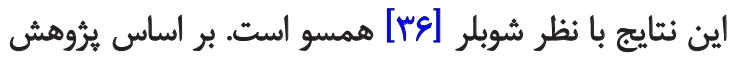

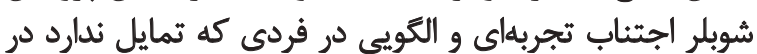


ميزان روشهاى تنظيم هيجان ناكارآهد بيرونى را تبيين كنيند

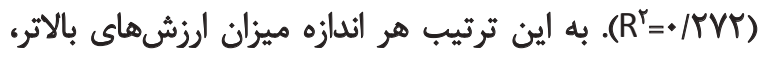

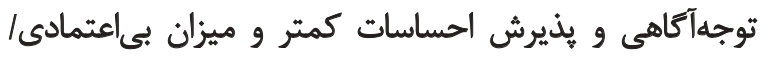

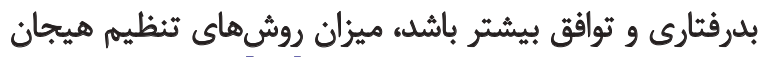

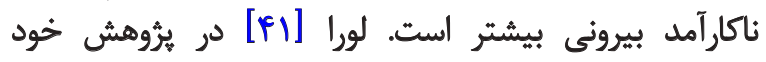

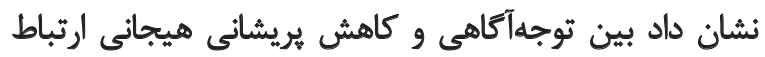

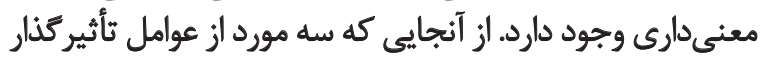

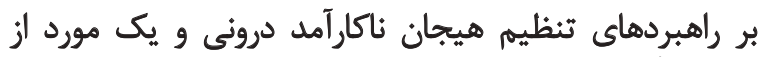

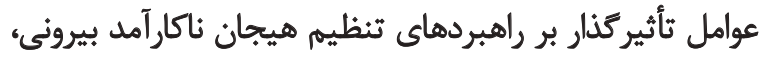

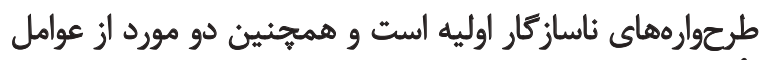

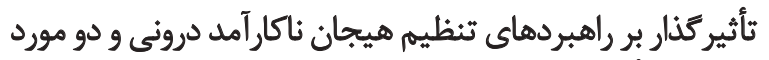

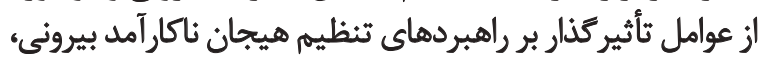

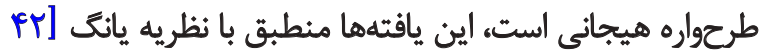
[T]،

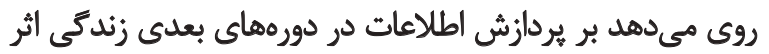

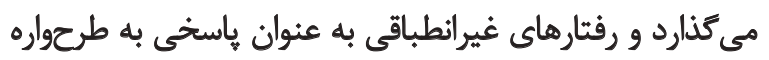

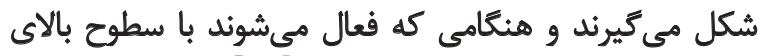

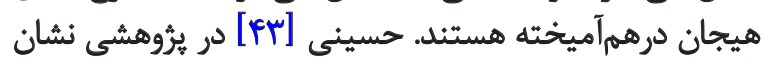

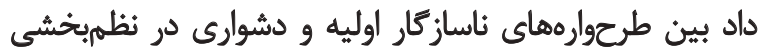
هيجانى رابطه معنى دارى وجود دارد. ناسنار اليه

اين يثروهش با محدوديتهايى مواجه بود كه مي توان به استفاده

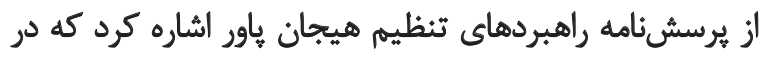

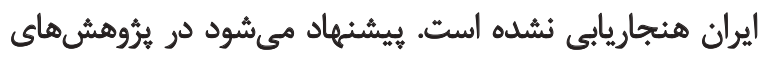

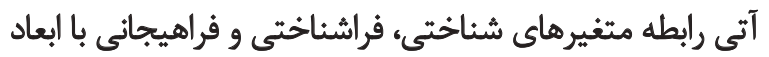

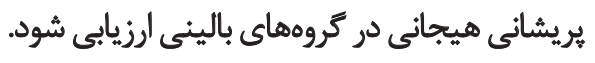

سياسكَّزارى

اين مقاله از باياننامه كارشناسى ارشد نويسنده اول در

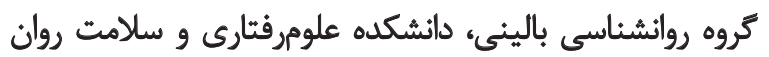

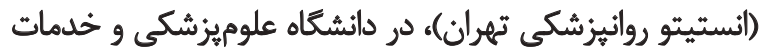

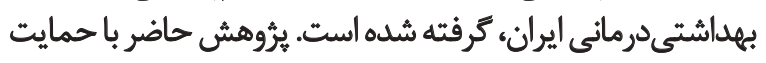

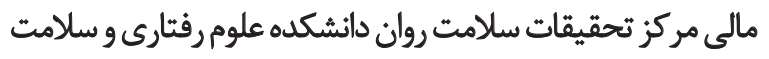

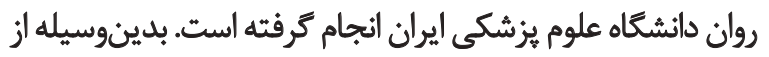

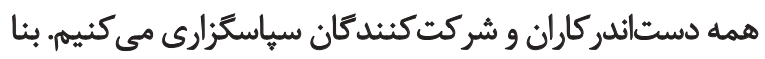

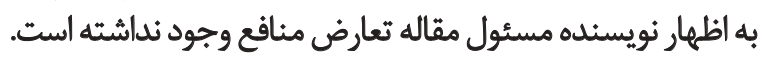




\section{References}

[1] Machado T. S, Pardal A. Emotional regulation \& adaptive learning strategies in Portuguese adolescents: A study with the regulation emotion questionnaire-2. Paper presented at The International Psychological Applications Conference and Trend. 26-28 April 2013; Madrid, Spain.

[2] Garner PW, Spears FM. Emotion regulation in low-income preschoolers. Social Development. 2000; 9(2):246-64. doi: 10.1111/1467-9507.00122

[3] Greenberg LS, Paivio SC. Working with emotions in psychotherapy. New York: Guilford Press; 2003.

[4] Thompson RA, Calkins SD. The double-edged sword: Emotional regulation for children at risk. Development and Psychopathology. 1996; 8(1):163. doi: 10.1017/s0954579400007021

[5] Gratz KL, Roemer L. Multidimensional assessment of emotion regulation and dysregulation: Development, factor structure, and initial validation of the difficulties in emotion regulation scale. Journal of Psychopathology and Behavioral Assessment. 2004; 26(1):41-54. doi: 10.1023/b.joba.0000007455.08539.94

[6] Gross JJ, Thompson RA. Emotion regulation: Conceptual foundations. New York: Guilford Press; 2007

[7] Narimani M, Ariapooran S, Abolghasemi A, Ahadi B. [Effectiveness of mindfulness-based stress reduction and emotion regulation training in the affect and mood of chemical weapons victims (Persian)]. Arak Medical University Journal. 2012; 15(2):107-18.

[8] Phillips KFV, Power MJ. A new self-report measure of emotion regulation in adolescents: The regulation of emotions questionnaire. Clinical Psychology \& Psychotherapy. 2007; 14(2):145-56. doi: $10.1002 /$ cpp.523

[9] Power M. Emotion-focused cognitive therapy. New Jersey: John Wiley \& Sons; 2010.

[10] Haghighat F, Tehranchi A, Dehkordian P, Rasoolzade Tabatabaei S K. [A study of the role of psychological factors and pain related variables in the prediction of emotional distress in patients with multiple sclerosis (Persian)]. Journal of Public Health Research. 2013; 6(4):1-11.

[11] McKay M, Wood JC, Brantley J. The dialectical behavior therapy skills workbook. Oakland: New Harbinger; 2007.

[12] Cisler JM, Olatunji BO, Feldner MT, Forsyth JP. Emotion regulation and the anxiety disorders: An integrative review. Journal of Psychopathology and Behavioral Assessment. 2009; 32(1):68-82. doi: 10.1007/s10862-009-9161-1

[13] Wells A. Metacognitive therapy for anxiety and depression. New York: Guilford Press. 2009.

[14] Wells A, Carter K. Further tests of a cognitive model of generalized anxiety disorder: Metacognitions and worry in GAD, panic disorder, social phobia, depression, and nonpatients. Behavior Therapy. 2001; 32(1):85-102. doi: 10.1016/s0005-7894(01)80045-9

[15] Gross JJ, Muñoz RF. Emotion regulation and mental health. Clinical Psychology: Science and Practice. 1995; 2(2):151-64. doi: 10.1111/j.1468-2850.1995.tb00036.x

[16] Enferadi H. [The relation between cognitive emotional regulation techniques with depression and anxiety in college students,
Comparison between clinical and non-clinical samples (Persian)] [MSc. thesis]. Tehran: Iran University of Medical Science; 2010.

[17] Gross JJ, John OP. Mapping the domain of expressivity: Multimethod evidence for a hierarchical model. Journal of Personality and Social Psychology. 1998; 74(1):170-91. doi: 10.1037/00223514.74.1.170

[18] Decker ML, Turk CL, Hess B, Murray CE. Emotion regulation among individuals classified with and without generalized anxiety disorder. Journal of Anxiety Disorders. 2008; 22(3):485-94. doi 10.1016/j.janxdis.2007.04.002

[19] Tabachnick BG, Fidell LS. Using multivariate statistics. Boston: Pearson Education; 2007.

[20] Leahy RL. A model of emotional schemas. Cognitive and Behavioral Practice. 2002; 9(3):177-90. doi: 10.1016/s10777229(02)80048-7

[21] Young JE, Klosko JS, Weishaar ME. Schema therapy: A practitioner's guide. New York: Guilford Press; 2003.

[22] Hayes SC. Mindfulness: method and process. Clinical Psychology: Science and Practice. 2003; 10(2):161-5. doi: 10.1093/clipsy/ bpg018

[23] Hayes SC, Luoma JB, Bond FW, Masuda A, Lillis J. Acceptance and commitment therapy: Model, processes and outcomes. Behaviour Research and Therapy. 2006; 44(1):1-25. doi: 10.1016/j. brat.2005.06.006

[24] Khanzadeh M, Saeediyan M, Hosseinchari M, Edrissi F. [Factor structure and psychometric properties of difficulties in emotional regulation scale (Persian)]. International Journal of Behavioral Sciences. 2012; 6(1):87-96.

[25] Young JE. Cognitive therapy for personality disorders: A schema-focused approach. Fruitville: Professional Resource Press; 1994.

[26] Oei TPS, Baranoff J. Young schema questionnaire: Review of psychometric and measurement issues. Australian Journal of Psychology. 2007; 59(2):78-86. doi: 10.1080/00049530601148397

[27] Gh A. [Normalization of young schema questionnaire: Short form (Persian)] [MSc. thesis]. Tehran: Allameh Tabataba'i University; 2006.

[28] Wells A, Cartwright-Hatton S. A short form of the metacognitions questionnaire: Properties of the MCQ-30. Behaviour Research and Therapy. 2004; 42(4):385-96. doi: 10.1016/s00057967(03)00147-5

[29] Abolghasemi A, Ahmadi M, Kiamarsi A. [The relationship of metacognition and perfectionism with psychological consequences in the addicts (Persian)]. Journal of Research in Behavioural Sciences. 2007; 5(2): 73-9.

[30] Sabet M. [The initial standardization of well's metacognitive test (Persian)]. Journal of Modern Thoughts in Education. 2010; 6(3): 27-50.

[31] Brown KW, Ryan RM. The benefits of being present: Mindfulness and its role in psychological well-being. Journal of Personality and Social Psychology. 2003; 84(4):822-48. doi: 10.1037/00223514.84.4.822

[32] Ghorbani N, Watson PJ, Weathington BL. Mindfulness in Iran and the United States: Cross-cultural structural complexity and 
parallel relationships with psychological adjustment. Current Psychology. 2009; 28(4):211-24. doi: 10.1007/s12144-009-9060-3

[33] Nejati VA, Amini RE, ZabihZadeh AB, Masoumi ME, Maleki G, Shoaie FA. [Mindfulness as effective factor in quality of life of blind veterans (Persian)]. Iranian Journal of War and Public Health. 2011;3(3):1-7.

[34] Bond FW, Hayes SC, Baer RA, Carpenter KM, Guenole N, Orcutt HK, et al. Preliminary psychometric properties of the acceptance and action questionnaire-II: A revised measure of psychological inflexibility and experiential avoidance. Behavior Therapy. 2011; 42(4):676-88. doi: 10.1016/j.beth.2011.03.007

[35] Abasi I, Fata L, Molodi R, Zarrabi H. [Psychometric properties of Persian version of acceptance and action questionnaire -iin (Persian)]. Journal of Psychological Models and Methods. 2012; 2(10):65-80.

[36] Mitmansgruber H, Beck TN, Höfer S, Schüßler G. When you don't like what you feel: Experiential avoidance, mindfulness and meta-emotion in emotion regulation. Personality and Individual Differences. 2009; 46(4):448-53. doi: 10.1016/j.paid.2008.11.013

[37] Kuyken W, Watkins E, Holden E, White K, Taylor RS, Byford $\mathrm{S}$, et al. How does mindfulness-based cognitive therapy work. Behaviour Research and Therapy. 2010; 48(11):1105-12. doi: 10.1016/j.brat.2010.08.003

[38] Roemer L, Lee JK, Salters-Pedneault K, Erisman SM, Orsillo SM, Mennin DS. Mindfulness and emotion regulation difficulties in generalized anxiety disorder: Preliminary evidence for independent and overlapping contributions. Behavior Therapy. 2009; 40(2):142-54. doi: 10.1016/j.beth.2008.04.001

[39] Deplus S, Billieux J, Scharff C, Philippot P. A mindfulnessbased group intervention for enhancing self-regulation of emotion in late childhood and adolescence: A pilot study. International Journal of Mental Health and Addiction. 2016; 14(5):775-90. doi: 10.1007/s11469-015-9627-1

[40] Kiken LG, Shook NJ. Mindfulness and emotional distress: The role of negatively biased cognition. Personality and Individual Differences. 2012; 52(3):329-33. doi: 10.1016/j.paid.2011.10.031

[41] Tang YY, Tang R, Posner MI. Mindfulness meditation improves emotion regulation and reduces drug abuse. Drug and Alcohol Dependence. 2016; 163:13-8. doi: 10.1016/j.drugalcdep.2015.11.041

[42] Leahy RL. Overcoming resistance in cognitive therapy. New York: Guilford Press. 2012

[43] Sabet Hosseini F, Salari Z. [The role of early maladaptive schemas and difficulties in emotion regulation in students' anxiety (Persian)]. Journal of Behavioral Sciences. 2014; 7(4):3-4. 